\title{
LAS PERCEPCIONES DE LA ÉTICA EN LOS TRABAJADORES DEL PUERTO DE MANZANILLO, COLIMA.
}

\author{
José Emanuel López Luna*, Aurelio Deniz Guizar**, Juan Alonzo Livas de la Garza***.
}

\author{
Las percepciones de la ética en los trabajadores del \\ puerto de Manzanillo, Colima. López-Luna J.E., Deniz- \\ Guizar A.,Livas-de la Garza J.A. Hitos de Ciencias \\ Económico Administrativas \\ 2011;17 (49):141-148.
}

\section{RESUMEN}

El presente artículo analiza la percepción que los trabajadores de Manzanillo (Colima) tienen respecto a la ética en tres conglomerados distintos: honestidad, dinámica laboral e interacción social; y está subdividido en tres apartados. En el primero, se presenta una demografía descriptiva, en donde se incluye información sobre la muestra en su conjunto; en el segundo, se realiza un análisis de conglomerados (mencionados anteriormente), en el que se asigna una calificación a los participantes en una escala del cero al diez de acuerdo a las respuestas aportadas; en el tercero, se realizan diversas pruebas de independencia entre variables, en el que se determina la intensidad y direccionalidad de las mismas.
The perception that workers at the Port of Manzanillo, Colima have regarding Ethics. López-Luna J.E., DenizGuizar A.,Livas-de la Garza J.A. Hitos de Ciencias Económico Administrativas 2011;17 (49):141-148.

\section{ABSTRACT}

This article analyzes the perceptions that workers in Manzanillo, Colima have in regard to ethics. The analysis has been organized into three different clusters: honesty, work-related dynamics, and social interaction. The article is then divided into three sections. The first one presents a descriptive demography where information of the sample as a whole is included; in the second section an analysis of the above mentioned conglomerates is performed and a rating is assigned to the participants on a scale of zero to ten according to their answers. In the third one, various tests of independence between variables are carried out, determining the intensity and directionality of them.

Palabras clave: Percepción. Ética. Trabajadores.

Key words: Perception. Ethics. Workers.

DIRECCIÓN PARA RECIBIR CORRESPONDENCIA: Correo electrónico: livasabc@ucol.mx

$\mathbf{M}$

anzanillo presenta un rostro multifacético, sus actividades económicas incluyen la pesca, la agricultura, la ganadería, el comercio, la industria y los servicios; no obstante, el sector de los servicios al Comercio Exterior emplea una parte importante de la fuerza laboral del puerto (aproximadamente el $7.84 \%$ de la Población Económicamente Activa (PEA) del municipio que se dedica esta actividad).

Debido a ello, resultaba importante realizar un estudio sobre la percepción que tienen los trabajadores respecto a la ética, con el fin de determinar si la cultura local ha comenzado a apropiarse de los valores intrínsecos que deben regir el comportamiento del hombre en sociedad.

A simple vista, podría parecer que realizar actos poco éticos (como sobornar a algún funcionario público para acortar tiempos o falsificar documentos para cumplir con requisitos de trámite) incrementaría la eficiencia y productividad de las empresas; sin embargo, diversos estudios han demostrado que se logra el efecto contrario

\footnotetext{
* Porfesor-Investigador de la Universidad de Colima, México.

** Porfesor-Investigador de la Universidad de Colima, México.

*** Porfesor-Investigador de la Universidad de Colima, México.
}

a nivel agregado. De acuerdo a la Encuesta sobre Gobernabilidad y Desarrollo Empresarial ${ }^{1}$, Ias empresas gastan alrededor del 6\% anual de sus ingresos en pagos extraoficiales para influir en las decisiones de los funcionarios públicos, ya sean éstos municipales, estatales o federales. La misma encuesta revela que las aduanas (una de las cuales está en Manzanillo y conecta de manera importante la cuenca del Pacífico con nuestro país), se encuentran en el tercer lugar de deshonestidad, al ser calificadas con un 7.1 (en una escala del cero al diez) respecto a ese indicador. En el estado de Colima, aproximadamente el $1 \%$ de las empresas realizan pagos extraoficiales a nivel federal, $0 \%$ a nivel estatal y $9 \%$ a nivel municipal.

De acuerdo al Informe Ejecutivo del Índice Nacional de Corrupción y Buen Gobierno de Transparencia Internacional ${ }^{2}$, durante 2007 se llevaron a cabo 197 millones de actos de corrupción en el uso de servicios públicos provistos por autoridades federales, estatales,

\footnotetext{
1 Centro de Estudios Económicos del Sector Privado, «Encuesta sobre gobernabilidad y desarrollo empresarial», CEESP, México, 2005.

${ }_{2}^{2}$ Transparencia Internacional, «Informe Ejecutivo del Índice Nacional de Corrupción y Buen Gobierno», 2007. Descargado de la red mundial el 17 de junio de 2009 (http://www.transparenciamexicana.org.mx/ documentos/INCBG/2007/Presentacion_INCBG_2007.pdf).
}

Fecha de recibido: 30 de abril de 2011 Fecha de aceptación: 14 de julio de 2011. 
municipales; así como concesiones y servicios provistos por particulares. esto es 375 actos de corrupción por minuto. A nivel internacional, dicho organismo ubica a México en el lugar 65 de 158 naciones respecto a actos de corrupción, a nivel país, Colima es el estado con menor corrupción (su índice es de 3.1, siendo 10 la media aritmética nacional), ocupando el primer lugar en una lista de 32 entidades; el Estado de México ocupa el último lugar al registrar 18.8 puntos en la misma escala.

No obstante, este sobresaliente primer lugar, en el estado de Colima se presentan una gran cantidad de actos ambiguos que podrían generar sesgos en el comportamiento normal de los actores económicos. En Manzanillo, por ejemplo, el personal de la aduana tiene autorizado recibir regalos de parte del sector privado, siempre y cuando no superen el $10 \%$ de su salario mensual; sin embargo, como parte del cruzamiento de datos, algunos de los funcionarios entrevistados manifestaron haber recibido obsequios que fácilmente superan ese tope. Dichos presentes quizá no tienen la finalidad directa de sobornar a quien los recibe, pero sí podrían motivarlos a corresponder mediante la concesión de favores que bajo condiciones normales de austeridad no otorgarían.

La Asociación de Agentes Aduanales del Puerto de Manzanillo, A.C. (AAAPUMAC) ha fungido como intermediario en épocas especiales (Navidad, por ejemplo), en las que los Agentes Aduanales realizan donaciones voluntarias de diversos artículos (maletines de piel, botellas de vino, bolígrafos de lujo, computadoras personales, canastas navideñas) para ser repartidas a los distintos trabajadores de las dependencias gubernamentales como agradecimiento por sus servicios; aunque el receptor es desconocido y suele realizarse por sorteo, ello podría generar también una ligera tendencia a ignorar el cumplimiento de las leyes aduanales a favor del gremio.

La presente investigación tiene como objetivo elaborar un diagnóstico al interior de las empresas, para determinar la facilidad con que ciertas normas éticasontológicas suelen ser violadas; al conocer esto, no debería sorprender que se proyecte externamente la vida interna de las interacciones socio-empresariales en los agentes económicos estudiados.

\section{FIGURA 1. EDAD DE LOS PARTICIPANTES.}

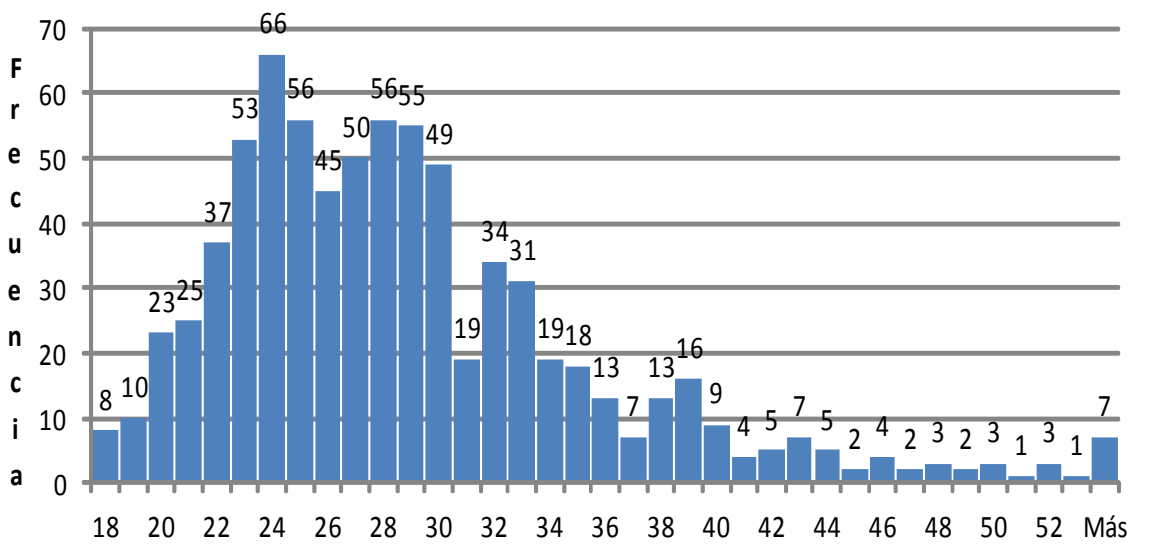

Fuente: elaboración propia.

\section{METODOLOGÍA.}

Para la recopilación de la información, se aplicaron 824 encuestas de manera aleatoria en 55 empresas relacionadas con los servicios al Comercio Exterior en Manzanillo durante 2008 y el primer semestre de 2009 (en la muestra se contemplaron las siguientes entidades: $100 \%$ de las empresas concesionarias del Gobierno Federal, $100 \%$ de las asociaciones civiles, $100 \%$ de las dependencias gubernamentales, $26 \%$ de las agencias navieras, $24 \%$ de las agencias aduanales, $22 \%$ de las empresas maniobristas, $20 \%$ de las empresas operadoras y $13 \%$ de las empresas de transportación terrestre). El $92.5 \%$ de esas empresas pertenece al sector privado y el $7.5 \%$ restante al sector público. De las 824 encuestas, sólo 771 superaron las pruebas de cruzamiento y coherencia de datos (se descartó el $6.43 \%$ de las mismas).

La encuesta contenía 61 preguntas en total, mediante la cual se obtenían datos básicos, como edad, género y escolaridad de la persona; así como datos relacionados con la frecuencia con que realizaba (o no) determinadas acciones que pudieran crear conflictos éticos. Dichas preguntas implicaban la existencia de un valor subyacente (por ejemplo: honestidad, responsabilidad, prudencia, lealtad, tolerancia). Los resultados se agruparon en tres categorías principales respecto al comportamiento de los encuestados, con la finalidad de responder las siguientes preguntas: ¿qué tan honesta es la persona?, ¿cómo es su dinámica laboral? y ¿cómo es su interacción social?, mismas que podrán ser observadas en la siguiente sección.

\section{RESULTADOS.}

Para la presentación de los resultados, se han diseñado tres secciones: demografía descriptiva, análisis de conglomerados y pruebas de independencia y direccionalidad, y en algunos casos se han eliminado los datos perdidos.

\section{Demografía descriptiva.}

El $81.1 \%$ de los participantes tiene 33 años de edad o menos (Figura 1) ${ }^{3}$, lo que evidencia una marcada tendencia del sector portuario por contar con una fuerza de trabajo joven. El rango en la edad de los encuestados es de 51 años, siendo 18 el mínimo estadístico y 69 el máximo. La edad promedio se situó en los 29 años de edad; la moda es 24.

El $55.1 \%$ pertenece al sexo masculino $y$ el $44.9 \%$ restante al femenino, lo cual, en términos de género, parece estar relativamente equilibrado.

Respecto al nivel de estudios, $1.3 \%$ cuenta con primaria solamente (véase Figura 2), $12.8 \%$ con secundaria, $31.5 \%$ con preparatoria, $0.3 \%$ con título de Profesional Asociado en Comercio Exterior, $52.8 \%$ con licenciatura y $1.3 \%$ con maestría (éstos no son datos absolutos y definitivos, dado que algunos participantes de nivel preparatoria afirmaban estar cursando una licenciatura). Se puede observar

${ }^{3}$ En la investigación no se detectó ningún menor de edad trabajando en este sector de la economía de Manzanillo. 
LÓPEZ-LUNA J.E., DENIZ-GUIZAR A., LIVAS-DE LA GARZA J.A.

mediante estos datos que el $85.9 \%$ de los encuestados cuentan con una educación media superior o superior, lo que implica la exigencia del medio aduanero por contar con personal altamente calificado.

En promedio, cada trabajador tiene 3.7 años laborando para la empresa. El rango es de casi 28 años (1 mes mínimo, 28 años máximo), siendo 2 años la moda (véase la Figura 3). El $81.8 \%$ de los participantes manifiesta tener 5 años o menos laborando para la empresa. Este fenómeno puede deberse a tres supuestos: 1. La empresa es relativamente joven (de reciente creación), 2. El medio aduanero absorbe inmediatamente trabajadores jóvenes conforme éstos emergen de los centros de estudio, o 3 . Altos índices de rotación del personal.

En cuanto al sueldo, el ingreso promedio mensual es de $\$ 8,415.89$. El sueldo mínimo registrado es de $\$ 600.00$, y el máximo es de $\$ 40,000.00$, siendo $\$ 7,000.00$ la moda (véase la Figura 4). El $78.4 \%$ de los participantes percibe $\$ 10,000.00$ mensuales o menos.

El $48.5 \%$ de los participantes afirma haber recibido algún curso o clase relacionada con la ética, aunque el $67.9 \%$ afirma que la familia ha influenciado más su comportamiento moral, por encima de cualquier educación académica recibida.

Respecto a la religión, $5.0 \%$ no manifiesta ninguna religión (véase la Figura 5), 88.7\% se manifiesta católico, $5.8 \%$ se manifiesta cristiano (protestante), y sólo el $0.5 \%$ se manifiesta Testigo de Jehová. Cabe resaltar que, de los católicos, sólo el $35.4 \%$ acude a los servicios religiosos semanalmente, mientras que la mayoría relativa $(40.0 \%)$ acude infrecuentemente (esporádicamente). En la sección de pruebas de independencia se determinará si la religión ejerce (o no) influencia respecto al comportamiento moral de los encuestados.

Para poder determinar si la persona tiene (o no) un concepto claro de lo que es la Ética, se le pidió una definición sobre la misma; el $67.9 \%$ cumplió con el cometido, mientras que el $32.1 \%$ restante afirmó no tener o no recordar una definición. En la Tabla 1 podemos observar el lugar en el que se ubican todas las definiciones proporcionadas; tan sólo el $28.4 \%$ de ellas puede ser considerada como aceptable, buena, muy buena o excelente. Lo que significa que, de la muestra total (771 participantes), sólo el $17.5 \%$ (135 participantes) pudo dar una definición por lo menos aceptable de lo que es la ética.

\section{Análisis de conglomerados.}

En esta sección se dará respuesta a las preguntas que se plantearon inicialmente: ¿qué tan honesta es la
FIGURA2. NIVEL EDUCATIVO.

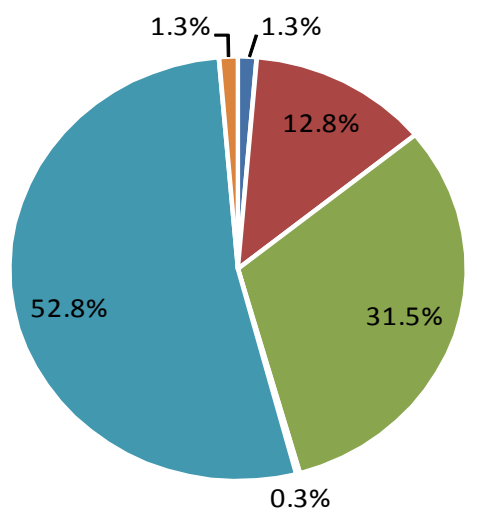

- Primaria

- Secundaria

- Preparatoria

- Profesional Asociado en Comercio Exterior

- Licenciatura

- Maestría

Fuente: elaboración propia.

\section{FIGURA 3. AÑOS LABORANDO PARA LA EMPRESA}

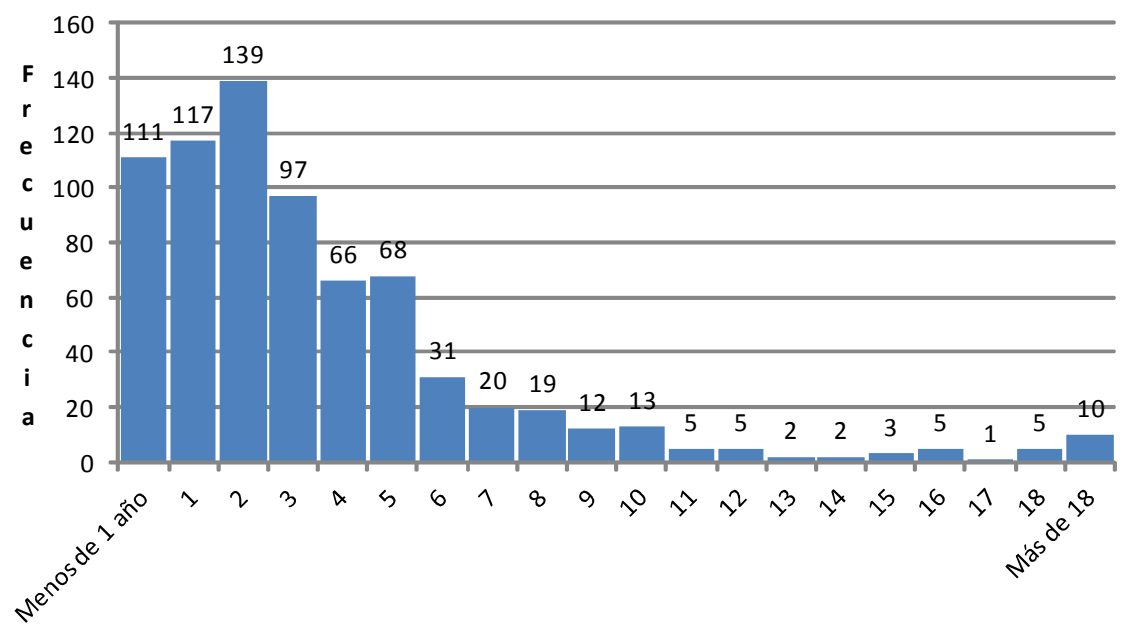

Fuente: elaboración propia.

Tiempo en años

FIGURA 4. INGRESO MENSUAL (SUELDO).

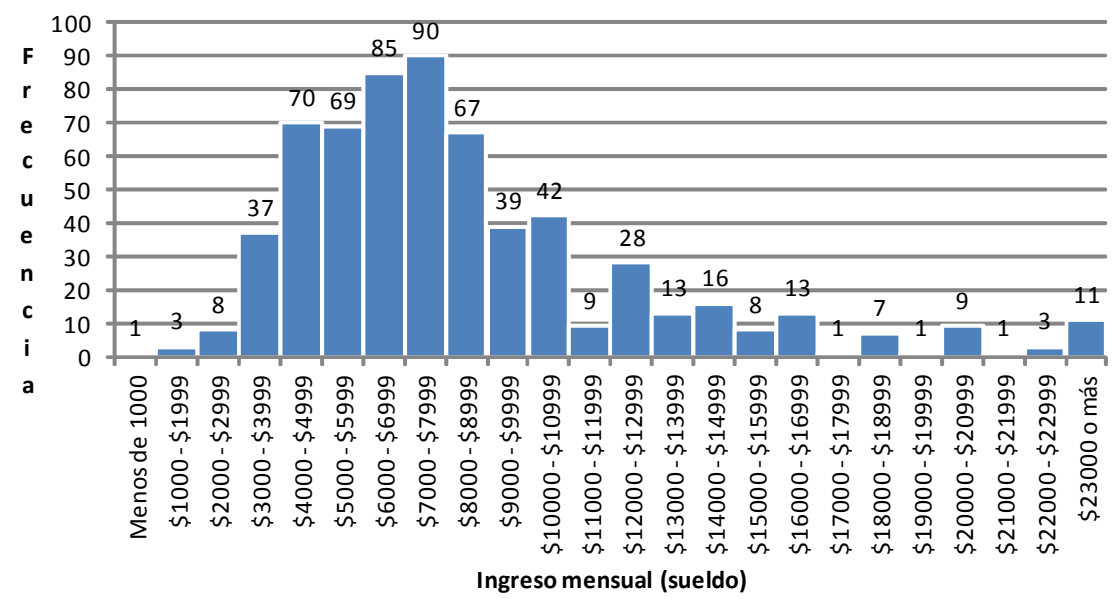

Fuente: elaboración propia.
Ingreso mensual (sueldo) 


\section{FIGURA5.RELIGIÓN DE LOS PARTICIPANTES}

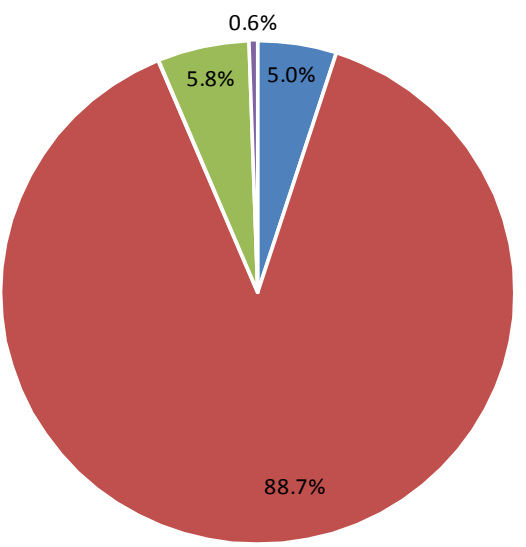

- Ninguna

- Catolicismo

$=$ Cristianismo

- Testigo de Jehová

Fuente: elaboración propia.

\section{TABLA I. MUESTRA EL LUGAR EN EL QUE SE UBICAN LAS DIFERENTES DEFINICIONES QUE LOS PARTICIPANTES PROPORCIONARON DURANTE LA INVESTIGACIÓN.}

\begin{tabular}{lcc}
\multicolumn{1}{c}{ Calificación } & Porcentaje & Acumulado \\
\hline Excelente & $9.3 \%$ & $9.3 \%$ \\
Muy buena & $4.6 \%$ & $13.9 \%$ \\
Buena & $4.0 \%$ & $17.9 \%$ \\
Aceptable & $10.5 \%$ & $28.4 \%$ \\
Regular & $53.5 \%$ & $81.9 \%$ \\
Equivocada & $18.1 \%$ & $100 \%$ \\
\hline Total & $100 \%$ &
\end{tabular}

Total

$100 \%$

Fuente: elaboración propia.

persona? (entendida como la «tendencia a no mentir, engañar o robar $\left.{ }^{4}\right)$, ¿cómo es su dinámica laboral? y ¿cómo es su interacción social? Para ello se utilizó el análisis de conglomerados de K-medias, asignando calificaciones en una escala del UNO al DIEZ a cada uno de los participantes, en donde el DIEZ representa el comportamiento ideal y el UNO el comportamiento más abyecto.

Ocho son las preguntas que evalúan la honestidad del participante; y tienen relación con la frecuencia con que ellos realizan los siguientes supuestos:

1. Realizar Ilamadas telefónicas personales a través del teléfono de la oficina.

$71.7 \%$ de los encuestados manifestó realizar llamadas telefónicas personales a través del teléfono de la oficina de vez en cuando o rara vez; $6.8 \%$ manifestó realizarlo siempre o generalmente. El resto nunca lo hacía.

2. Consumir tiempo de trabajo en actividades distintas a las laborales (como dialogar con los compañeros, leer el periódico, escuchar música, chatear, maquillarse,

${ }^{4}$ Grover, L.D. «The truth, the whole truth, and nothing but the truth: The causes and management of workplace lying" Academy of Management Executive. Vol. 19 (2). P. 148. entre otras).

$70.9 \%$ de los encuestados manifestó consumir tiempo de trabajo en actividades distintas a las laborales de vez en cuando o rara vez; $6.6 \%$ manifestó realizarlo siempre o generalmente. El resto nunca lo hacía.

3. Utilizar el equipo de cómputo para actividades distintas a las laborales.

$66.3 \%$ de los encuestados manifestó utilizar el equipo de cómputo para actividades distintas a las laborales de vez en cuando o rara vez; $6.1 \%$ manifestó realizarlo siempre o generalmente. El resto nunca lo hacía.

4. Mentir en cosas sin importancia.

$44.9 \%$ de los encuestados manifestó mentir en cosas sin importancia de vez en cuando o rara vez; $2.5 \%$ manifiesta realizarlo siempre o generalmente. El resto nunca lo hacía.

5. Extraer cosas de poco valor de la oficina (como hojas, plumas, lápices, entre otros).

$19.6 \%$ de los encuestados manifestó extraer cosas de poco valor de la oficina de vez en cuando o rara vez; $0.7 \%$ manifestó realizarlo siempre o generalmente. El resto nunca lo hacía.

6. Alterar o falsificar documentos para facilitar el trabajo.

$15.2 \%$ de los encuestados manifestó alterar o falsificar documentos para facilitar el trabajo de vez en cuando o rara vez; $1.2 \%$ manifestó realizarlo siempre o generalmente. El resto nunca lo hacía.

7. Apropiarse de las ideas de otros y presentarlas como nuestras para obtener reconocimiento.

$5.0 \%$ de los encuestados manifestó apropiarse de las ideas de otros y presentarlas como propias para obtener reconocimiento de vez en cuando o rara vez; $0.4 \%$ manifestó realizarlo generalmente. El resto nunca lo hacía.

\section{Llevarse los clientes consigo cuando se cambia de} trabajo.

$4.6 \%$ de los encuestados manifestó llevarse consigo los clientes cuando se cambia de trabajo de vez en cuando o rara vez; $1.4 \%$ manifestó realizarlo siempre o generalmente. El resto nunca lo hacía.

En la Tabla II, muestra la calificación de cada uno de los participantes en la escala mencionada al principio de este apartado. En términos generales, sólo el $3.6 \%$ de la muestra puede ser calificada como totalmente honesta, mientras que el resto comete alguna de las ocho acciones anteriores con la frecuencia descrita.

Como puede analizarse, podemos percatarnos los encuestados realizan un «robo discreto» en la empresa (disponer del tiempo y los recursos de la misma para fines distintos a los planeados); dicho robo se ve reflejado en los supuestos 1, 2, 3 y 5; de entre ellos, el que más sobresale por su frecuencia, es el uso indiscriminado del teléfono para efectos personales. Con el uso de software, las compañías ahora pueden asignar a cada empleado un código de acceso a línea externa, mismo que contiene el alcance con el que el empleado podrá acceder a llamadas locales, celulares, nacionales o internacionales. Sin embargo, y debido a las características de la cultura mexicana, dicho mecanismo resulta insuficiente, dado que los empleados pueden fácilmente digitar su código y permitirle a otra persona una llamada personal (como un «favor»), o bien, revelarles su código directamente para 
TABLAII. MUESTRALACALIFICACION QUE OBTUVIERON LOS 771 PARTICIPANTES EN TÉRMINOS DE HONSTIDAD EN UNA ESCALA DEL UNO AL DIEZ, DONDE DIEZ REPRESENTA ABSOLUTAHONESTIDAD.

\begin{tabular}{cccc}
$\begin{array}{c}\text { Calificación en } \\
\text { términos de } \\
\text { honestidad }\end{array}$ & $\begin{array}{c}\text { Frecuencia } \\
\text { (encuestados) }\end{array}$ & Porcentaje & $\begin{array}{c}\text { Porcentaje } \\
\text { acumulado }\end{array}$ \\
\hline 10 & 28 & 3.6 & 3.6 \\
9 & 346 & 44.9 & 48.5 \\
8 & 82 & 10.6 & 59.1 \\
7 & 95 & 12.3 & 71.4 \\
6 & 64 & 8.3 & 79.7 \\
5 & 29 & 3.8 & 83.5 \\
4 & 36 & 4.7 & 88.2 \\
3 & 70 & 9.1 & 97.3 \\
2 & 18 & 2.3 & 99.6 \\
1 & 3 & 0.4 & 100 \\
\hline Total & 771 & 100 &
\end{tabular}

Fuente: elaboración propia.

que ellos puedan hacer las llamadas que deseen. Lo más recomendable sería sensibilizar a los empleados respecto al menoscabo que se realiza con ello en las finanzas de la empresa, y que podría poner en riesgo sus puestos de trabajo. De igual forma sería recomendable analizar otras opciones para reducir los gastos de telefonía, como el uso de voz sobre protocolo de internet (VolP por sus siglas en inglés), que aprovecha las ventajas públicas que la red mundial tiene sobre las redes privadas de las compañías telefónicas.

Los supuestos 2, 3 y 5 se materializan cuando existe una insuficiente supervisión del empleado; si bien es cierto que una «sobre observación» es contraproducente, una supervisión adecuada podría incrementar la eficiencia y rentabilidad de la compañía. Algunos quizá argumenten que el realizar las acciones mencionadas anteriormente les asegura el cumplimiento del principio básico de reciprocidad, es decir, la empresa está obligada a otorgar ciertos beneficios a los empleados que la hacen ser productiva, compensando con ello las horas que laboran de más, así como los distintos sacrificios en los que se incurre para concluir con el trabajo a tiempo. Sin embargo los datos muestran que el $69.5 \%$ de ellos labora ocho horas diarias o menos y, más aún, el $79.4 \%$ de los encuestados cuenta diariamente con tiempo suficiente para salir a comer, por lo que el argumento de la reciprocidad podría quedar sin validez.

En cuanto a la dinámica laboral, seis son las preguntas que evalúan dicho rubro, y analizan la frecuencia con que ellos realizan los siguientes supuestos:

1. Llegar tarde al trabajo.

$70.8 \%$ de los encuestados manifestó llegar tarde al trabajo de vez en cuando o rara vez; 4.5\% manifestó realizarlo siempre o generalmente. El resto nunca lo hacía.

2. Salir antes de la hora señalada.

$43.4 \%$ de los encuestados manifestó salir antes de la hora señalada de vez en cuando o rara vez; $3.0 \%$ manifestó realizarlo siempre o generalmente. El resto nunca lo hacía.

\section{Revelarle al jefe el contenido de las conversaciones}

de otros.

$16.2 \%$ de los encuestados manifestó revelarle al jefe el contenido de las conversaciones de otros de vez en cuando o rara vez; $1.6 \%$ manifestó realizarlo siempre o generalmente. El resto nunca lo hacía.

\section{Hablar bien del jefe con los compañeros para obtener posteriormente algún favor.}

$13.8 \%$ de los encuestados manifestó hablar bien del jefe con los compañeros para obtener posteriormente algún favor de vez en cuando o rara vez; $4.2 \%$ manifestó realizarlo siempre o generalmente. El resto nunca lo hacía.

\section{Avisarle al jefe cuando se vaya a faltar al trabajo o a llegar tarde. \\ $8.9 \%$ de los encuestados manifestó hacerlo rara vez o nunca; el $17.3 \%$ lo hace generalmente o de vez en cuando. El resto lo hacía siempre.}

6. Crearse enfermedades o provocarse accidentes para faltar al trabajo de manera justificada.

Sólo $2.9 \%$ de los encuestados manifestó hacerlo de vez en cuando o rara vez; $0.4 \%$ manifestó realizarlo siempre o generalmente. El resto nunca lo hacía.

En la Tabla III muestra la calificación de cada uno de los participantes en la escala mencionada al principio de este apartado. El análisis de K-medias reveló que 12 de los 771 participantes no podían ser clasificados en alguno de los grupos escalares, dado que sus respuestas escapaban a las premisas del ordenamiento. En términos generales, se puede afirmar que sólo el $12.6 \%$ de los encuestados manifiesta una excelente dinámica laboral. El resto suele cometer con cierta frecuencia una o más de las faltas implicadas en las seis preguntas mencionadas anteriormente, y que reducen la eficiencia y productividad al interior de la empresa.

Aunque algunos de los supuestos incluidos en la dinámica laboral podrían pertenecer también al ámbito de la honestidad, se ha decidido dejarlos aquí ya que encajan mejor con el perfil de referencia.

La cuestión de la puntualidad es, en tiempos modernos, una de las brechas de calidad más remarcadas de la

TABLA III. MUESTRA LA CALIFICACIÓN QUE OBTUVIERON LOS 771 PARTICIPANTES EN TÉRMINOS DE DINÁMICA LABORAL EN UNA ESCALA DEL UNO AL DIEZ, DONDE EL DIEZ REPRESENTA LOIDEAL.

\begin{tabular}{cccc}
$\begin{array}{c}\text { Calificación en } \\
\text { términos de } \\
\text { dinámica laboral }\end{array}$ & $\begin{array}{c}\text { Frecuencia } \\
\text { (encuestados) }\end{array}$ & Porcentaje & $\begin{array}{c}\text { Porcentaje } \\
\text { acumulado }\end{array}$ \\
\hline 10 & 97 & 12.6 & 12.6 \\
9 & 248 & 32.2 & 44.8 \\
8 & 214 & 27.8 & 72.6 \\
$\mathbf{7}$ & 59 & 7.6 & 80.2 \\
$\mathbf{6}$ & 24 & 3.1 & 83.3 \\
$\mathbf{5}$ & 25 & 3.2 & 86.5 \\
$\mathbf{4}$ & 2 & 0.3 & 86.8 \\
$\mathbf{3}$ & 3 & 0.4 & 87.2 \\
$\mathbf{2}$ & 70 & 9.1 & 96.3 \\
$\mathbf{1}$ & 17 & 2.2 & 98.5 \\
Sin calificación & 12 & 1.5 & 100 \\
\hline Total & $\mathbf{7 7 1}$ & $\mathbf{1 0 0}$ &
\end{tabular}

Fuente: elaboración propia. 
cultura mexicana. El supuesto uno corrobora dicha afirmación. Hise, Solano-Méndez y Gresham ${ }^{5}$ hacen quince recomendaciones cuando se negocia con los mexicanos, una de ellas es "estar dispuestos a ser flexibles respecto a la hora en que dan inicio las reuniones», justamente por la premisa mencionada. Sorohan6 dice que los mexicanos podemos ser puntuales, pero que nuestro reloj funciona de manera diferente a otros países, por lo que nuestras contrapartes deberán llegar a tiempo y estar dispuestos a esperar, sin hacer posteriormente ningún comentario sobre ello.

Respecto a la interacción social que impera en los lugares de trabajo, la muestra se comportó conforme a los siguientes supuestos:

\section{Decirle al otro lo que piensas, sin importar cómo se sienta por ello. \\ $50.1 \%$ de los encuestados manifestó hacerlo de vez en cuando o rara vez; $20.9 \%$ manifestó realizarlo siempre o generalmente. El resto nunca lo hacía.}

2. Ignorar permanentemente a quien nos caiga mal. $41.6 \%$ de los encuestados manifestó ignorar a quien les cae mal de vez en cuando o rara vez; $13.4 \%$ manifestó hacerlo siempre o generalmente. El resto nunca lo hacía.

\section{Remarcar los defectos de los compañeros.}

$38.7 \%$ de los encuestados manifestó remarcar los defectos de los compañeros de vez en cuando o rara vez; $2.7 \%$ manifiesta hacerlo siempre o generalmente. El resto nunca lo hacía.

4. Tener preferencias por determinados compañeros. $35.8 \%$ de los encuestados manifestó tener preferencias por determinados compañeros de vez en cuando o rara vez; $17.3 \%$ manifestó hacerlo siempre o generalmente. El resto nunca lo hacía.

\section{Echarle la culpa a los demás por los problemas que surjan de improviso. \\ $16.0 \%$ de los encuestados manifestó realizarlo de vez en cuando o rara vez; $0.7 \%$ manifestó hacerlo siempre o generalmente. El resto nunca lo hacía.}

\section{Tratar a los compañeros de puestos inferiores de manera diferente a como se trata al jefe. \\ $15.6 \%$ de los encuestados manifestó realizarlo de vez en cuando o rara vez; $7.7 \%$ hacerlo manifestó siempre o generalmente. El resto nunca lo hacía.}

\section{Elaborar chismes o causar intriga entre los compañeros. \\ $11.2 \%$ de los encuestados manifestó elaborar chismes o causar intriga entre los compañeros de vez en cuando o rara vez; $0.5 \%$ lo hace siempre o generalmente. El resto nunca lo hacía.}

8. Tratar de crear un ambiente de trabajo placentero. $5.5 \%$ de los encuestados manifestó que rara vez o nunca tratan de crear un ambiente de trabajo placentero; $46.9 \%$ trata de vez en cuando o generalmente. El resto lo hacía siempre.

\footnotetext{
${ }^{5}$ Hise, Richard; Solano-Méndez, Roberto y Gresham, Larry, "Doing Business in Mexico" Thunderbird International Business Review, Vol. 45(2), pp. 211-224.

${ }^{6}$ Sorohan, Erica, In practice, Training \& Development, Vol. 48 (7), p12.
}

9. Fingir que se está ocupado para no ayudar a alguien más.

$3.3 \%$ de los encuestados manifestó que generalmente o de vez en cuando fingen que están ocupados para no ayudarle a alguien más; 96.7\% manifiesta fingir rara vez o nunca.

10. Tratar a todos con respeto.

$1.4 \%$ de los encuestados manifestó que nunca o rara vez trata a todos con respeto; $25.8 \%$ manifestó hacerlo de vez en cuando o generalmente. El resto lo hacía siempre.

En este caso, sólo el 3.9\% de los encuestados manifiesta tener una excelente interacción social. El análisis de Kmedias revela que 30 participantes (3.9\% de la muestra) no puede ser calificada, debido a que escapa a las premisas del ordenamiento.

La interacción social en Manzanillo posee la calificación más pobre de los tres aspectos analizados. Mientras que en dinámica laboral $72.6 \%$ de los encuestados están por encima del 7 y en honestidad el $59.1 \%$ se ubica por encima de este valor, en interacción social sólo el $40.6 \%$ lo supera. En estudios posteriores convendría analizar si los reclutadores consideran este aspecto al momento de realizar las contrataciones, y determinar anticipadamente si el futuro trabajador posee las cualidades necesarias, no sólo para interrelacionarse adecuadamente con sus compañeros, sino para motivarlos a través del ejemplo. Sin duda, un ambiente de trabajo placentero es primordial para la realización satisfactoria de los objetivos de la compañía; incluso este factor puede llegar a considerarse como "otro salario», gracias al cual los empleados se sienten motivados a identificarse con la empresa y a actuar en consecuencia; este "salario» responde a elementos poco mensurables y la mayoría de los casos escapa al control de los administradores.

\section{Pruebas de independencia y direccionalidad.}

En esta sección se presentan los resultados de las pruebas de independencia y direccionalidad ( $d$ de Somers) realizadas a los conglomerados anteriores (honestidad, dinámica laboral e interacción social) respecto las siguientes 17 variables categóricas:

\section{Género}

2. Edad

3. Nivel educativo

4. Definición de ética

5. Horas que la persona labora diariamente

6. Horas efectivas para comidas

7. Años de permanencia en la empresa

8. Inquietud por cambiar de trabajo

9. Grado de satisfacción laborando para la empresa

10. Posibilidades de ascenso

11. Sector al que pertenece la empresa

12. Sueldo (ingreso mensual)

13. Grado de satisfacción con el sueldo

14. Razón sueldo - nivel de vida digno

15. Asistencia a algún curso sobre Ética

16. Religión que profesa

17. Asistencia a servicios religiosos (si es el caso)

Los resultados se presentan a continuación:

Honestidad.

En la Tabla IV se muestra los resultados de las pruebas de direccionalidad e independencia para el conglomerado honestidad. Las asociaciones que han rechazado la hipótesis nula han sido remarcadas. 
TABLA IV. RESULTADOS DE LAS PRUEBAS DE INDEPENDENCIA Y DIRECCIONALIDAD RESPECTO AL CONGLOMERADO HONESTIDAD.

\begin{tabular}{|c|c|c|}
\hline Variable & $d$ de Somers & $\begin{array}{c}\text { Significancia } \\
\text { aproximada }\end{array}$ \\
\hline 1. Género $(1 \rightarrow$ Hombre, $2 \rightarrow$ Mujer $)$ & -0.003 & 0.914 \\
\hline 2. Edad & 0.050 & 0.052 \\
\hline 3. Nivel educativo & -0.005 & 0.869 \\
\hline 4. Definición de Ética & -0.047 & 0.236 \\
\hline 5. Horas que la persona labora diariamente & 0.013 & 0.688 \\
\hline $\begin{array}{l}\text { 6. Horas efectivas para comidas } \\
\qquad(1 \rightarrow \text { Sí, } 2 \rightarrow \text { No })\end{array}$ & -0.030 & 0.325 \\
\hline 7. Años de permanencia en la empresa & 0.082 & 0.004 \\
\hline $\begin{array}{l}\text { 8. Inquietud por cambiar de trabajo } \\
\qquad(1 \rightarrow \text { Sí, } 2 \rightarrow \text { No })\end{array}$ & 0.054 & 0.087 \\
\hline $\begin{array}{l}\text { 9. Satisfacción por laborar en esta empresa } \\
\qquad(1 \rightarrow \text { Sí, } 2 \rightarrow \text { No })\end{array}$ & -0.056 & 0.056 \\
\hline 10. Posibilidades de ascenso & 0.028 & 0.361 \\
\hline $\begin{array}{l}\text { 11. Sector al que pertenece la empresa } \\
(1 \rightarrow \text { Público, } 2 \rightarrow \text { Privado })\end{array}$ & -0.085 & 0.000 \\
\hline 12. Sueldo (ingreso mensual) & -0.015 & 0.605 \\
\hline $\begin{array}{l}\text { 13. Satisfacción respecto al sueldo } \\
(1 \rightarrow \text { Sí, } 2 \rightarrow \text { No })\end{array}$ & -0.083 & 0.009 \\
\hline $\begin{array}{l}\text { 14. Razón sueldo - nivel de vida digno } \\
(1 \rightarrow \text { Positivo, } 2 \rightarrow \text { Negativo })\end{array}$ & -0.032 & 0.319 \\
\hline $\begin{array}{l}\text { 15. Asistencia a algún curso sobre Ética } \\
(1 \rightarrow \text { Sí, } 2 \rightarrow \text { No })\end{array}$ & -0.096 & 0.002 \\
\hline 16. Religión & 0.050 & 0.086 \\
\hline $\begin{array}{l}\text { 17. Asistencia a servicios religiosos } \\
\text { (si es el caso) }\end{array}$ & -0.091 & 0.003 \\
\hline
\end{tabular}

Fuente: elaboración propia.

Si bien, la direccionalidad es relativamente moderada, de la Tabla IV, se puede inferir que los trabajadores que muestran un comportamiento más honesto son los siguientes:

a) Quienes tienen más años de antigüedad.

b) Los del sector público.

c) Quienes están satisfechos con su sueldo.

d) Quienes han asistido a un curso sobre ética.

e) Quienes frecuentan más los servicios religiosos.

De éstas cinco, sólo la variable "asistir a cursos de ética» es la única que podría depender completamente de la empresa, dado que a través de la capacitación y educación continua se puede sensibilizar a los empleados para que éstos manifiesten un comportamiento transparente, lo cual podría contraer otros beneficios para el ambiente de trabajo, la eficiencia y la rentabilidad de la entidad.

Obsérvese, que el nivel educativo no presenta una relación de dependencia con la honestidad (cualquiera que sea el grado académico de la persona, no hay forma de presuponer su comportamiento ético). En la misma situación están las once variables restantes. Considerar la variable "sueldo» como otro ejemplo; en base al resultado del análisis se puede inferir que es independiente del conglomerado analizado; es decir, este estudio resalta que lo importante no es cuánto gana la persona, sino si ésta se siente satisfecha con ese ingreso (en otras palabras, ganar más no motiva necesariamente a la persona a ser más honesta).

\section{Dinámica laboral.}

En cuanto a la dinámica laboral, las únicas dos variables que muestran una dependencia moderada con ella son el género y el sueldo. Las mujeres, de acuerdo al análisis, presentan una mejor dinámica laboral que los hombres. En el caso de la variable "sueldo" se presenta una direccionalidad negativa; ello significa que, conforme mayor es el sueldo de la persona, su dinámica laboral se reduce significativamente.

\section{Interacción social.}

El análisis revela que las personas que presentan una mejor dinámica laboral son las siguientes:

a) Quienes aportaron una mejor definición de ética.

b) Quienes se sienten satisfechas laborando para la empresa.

c) Quienes asisten con más frecuencia a los servicios religiosos (misa, asamblea, reuniones).

Puede observarse que las primeras dos variables se encuentran efectivamente dentro del rango de influencia de la empresa. Tener una mejor definición de la ética podría motivar a la persona a interrelacionarse mejor con sus compañeros de trabajo; lograr esto podría depender de la asistencia a cursos de capacitación y sensibilización (al igual que en el conglomerado de la honestidad).

Lograr que un empleado se sienta satisfecho laborando para la empresa podría implicar la conjunción de una serie de variables poco mensurables (posicionamiento social de la empresa, políticas de calidad integral, políticas de selección de personal, ergonomía del lugar de trabajo, tiempos de traslado), por lo que resulta recomendable que las empresas realicen entrevistas regulares a los trabajadores a este respecto con la finalidad de monitorear este nivel de satisfacción.

El análisis de dependencia y direccionalidad nos da una idea clara de la interacción entre las variables y los conglomerados de agrupación. Desafortunadamente la empresa sólo puede influir positiva y directamente en una de las quince variables (asistir a cursos sobre ética) para lograr que sus empleados muestren un comportamiento más ético. El resto de las variables escapan al rango de influencia de la empresa o dependen de una conjunción de factores poco controlables. 


\section{CONCLUSIONES Y RECOMENDACIONES.}

El puerto de Manzanillo demanda fuerza de trabajo joven con un alto nivel académico para la prestación de servicios al Comercio Exterior. Los trabajadores en este sector perciben un sueldo menor a los $\$ 9,000.00$ (mensuales promedio), y aunque sólo el $35.4 \%$ de los encuestados manifestó que ese ingreso no les aseguraba un nivel de vida digno, poco más del $56 \%$ de los trabajadores se muestra insatisfecho con él.

De la muestra, sólo el $17.6 \%$ pudo dar una definición aceptable (por lo menos) de lo que era la ética. El análisis de dependencia y direccionalidad arrojó un resultado positivo en el conglomerado de la interacción social; es decir, conforme mejor definiera la ética el participante, su interacción social mejoraba.

Profesar una determinada religión no afecta el comportamiento de la persona; es la frecuencia con que la ésta atiende los servicios religiosos la que lo modifica. Esta última variable (frecuencia) mostró una dependencia moderada respecto al conglomerado honestidad e interacción social; en otras palabras, mientras mayor sea la frecuencia con que una persona atiende los servicios religiosos, mayor será su honestidad y tendrá una mejor interacción social en el trabajo.

En el primer conglomerado, sólo un $3.6 \%$ de la muestra se manifiesta completamente honesta. La asistencia a cursos de Ética podría modificar este patrón, ya que el análisis revela una dependencia positiva moderada al respecto. Ésta sería la única estrategia al alcance de la empresa para influir en el comportamiento de sus empleados, ya que las demás variables analizadas manifiestan una marcada independencia con el conglomerado o escapan al ámbito de influencia directa. De igual forma, en la medida en que los empleados permanezcan en la empresa, éstos manifestarán un comportamiento más honesto, por lo que deberá esforzarse por reducir el índice de rotación de personal mediante la implementación de estrategias que le permitan al empleado sentirse motivado e identificado con la compañía. Otra de las razones de dependencia interesantes que arrojó el análisis, es que los empleados del sector público manifiestan un comportamiento más honesto que los del sector privado. El sueldo, de acuerdo al estudio, resulta irrelevante en términos de este primer conglomerado, es decir, no hay una relación directa entre ambas variables (ganar más no significa ser más honesto. De igual forma, ganar menos no significa ser menos honesto); sin embargo, la satisfacción que experimenta el empleado sobre su sueldo, sí manifiesta una dependencia positiva moderada. La empresa, por lo tanto, debe tomar en cuenta el hecho que un trabajador no se sienta satisfecho con lo que percibe. En este caso, más allá de la magnitud, es la percepción que el trabajador tiene sobre su ingreso la que lo motiva a ceñir su comportamiento a principios éticos.

En el conglomerado de la dinámica laboral, la variable sueldo sí presenta una relación inversa moderada (ganar más empeora la dinámica laboral). En términos generales, sólo el $12.6 \%$ de los encuestados manifiesta una excelente dinámica laboral, siendo las mujeres quienes muestran un mejor indicador al respecto. La brecha de calidad aquí es la puntualidad, dado que un porcentaje muy alto de los encuestados manifiesta llegar tarde al trabajo con cierta frecuencia.

En cuanto a la interacción social, ésta presenta las mayores áreas de oportunidad, ya que tiene la calificación más baja de los tres conglomerados. Una de las tres variables que muestra dependencia positiva es la satisfacción del empleado por laborar en la empresa, lo que debe impulsar a mantener e incrementar ese indicador, a través de mecanismos específicos que aseguren su permanencia en la compañía.

El análisis general de dependencia y direccionalidad revela que el único instrumento con el que cuentan las empresas para mejorar el comportamiento integral del empleado son los cursos de sensibilización, en donde se traten temas relacionados con la ética; no está de más recomendar una excelente política de selección de personal, ya que esto asegurará un sano ambiente de trabajo. El departamento de Recursos Humanos, por ende, tiene a cuestas una tarea de suma importancia, ya que debe filtrar a los candidatos que manifiesten un comportamiento deshonesto, con una débil dinámica laboral o una inadecuada interacción social desde el principio. Y aunque al trabajador sólo se le pueda obligar a cumplir con las funciones que el análisis de su puesto le obliga, motivar el cumplimiento de diversos aspectos básicos que sustentan el equilibrio de los grupos sociales asegura que el ambiente de trabajo en su conjunto sea una plataforma para la realización de la persona.

\section{REFERENCIAS}

Centro de Estudios Económicos del Sector Privado. (2005). Encuesta sobre gobernabilidad y desarrollo empresarial. CEESP, México.

Grover, L. D. The truth, the whole truth and nothing but the truth: The causes and management of workplace lying. Academy of Management Executive. Vol. 19 (2).

Hise, Richard; Solano, Roberto y Gresham, Larry. Doing Business in Mexico. Thunderbird International Business Review, Vol. 45 (2).

Sorohan, Erica. In practice. Training \& Development. Vol. 48 (7).

Transparencia Internacional (17 de junio de 2009). Informe Ejecutivo del Índice Nacional de Corrupción y Buen Gobierno. Recuperado de (http://www.transparenciamexicana.org.mx/ documentos/INCBG/2007/Presentacion_INCBG_2007.pdf) 